\title{
The impact of a sense of coherence in employees with chronic pain
}

\author{
Monica Lillefjell ${ }^{\mathrm{a}, \mathrm{b}, *}$, Klara Jakobsen ${ }^{\mathrm{a}}$ and Linda Ernstsen ${ }^{\mathrm{c}}$ \\ ${ }^{a}$ Research Centre for Health Promotion and Resources HiST/NTNU, Department of Occupational Therapy, Faculty \\ of Health Education and Social Work, Sфr-Trøndelag University College, Trondheim, Norway \\ ${ }^{\mathrm{b}}$ Department of Social Work and Health Science, Norwegian University of Science and Technology, NTNU, \\ Trondheim, Norway \\ ${ }^{\mathrm{c}}$ Faculty of Nursing, Sфr-Trфndelag University College, Trondheim, Norway
}

Received 24 January 2013

Accepted 5 October 2013

\begin{abstract}
.
BACKGROUND: Although a strong sense of coherence, described as the dimensions of a person's response to a stressful situation, improves the prospects of coping with health problems, more knowledge is required about the association between initial anxiety levels and physical functioning after rehabilitation in relation to individuals' own experiences of their healthresources.

OBJECTIVE: To investigate if the associations between anxiety, pain, and functional health status differ with a strong and weak sense of coherence in employees with chronic musculoskeletal pain in rehabilitation.

METHODS: One thousand six hundred and sixty-five employees (mean age $=45.2 / S D=9.1$ ) were included in the study. Self-reported sense of coherence (SOC), personal characteristics, anxiety, functional status, and pain were collected at the start (T1) and at the end of the rehabilitation period (12 weeks) (T2). Based on validated cut-off values the employees were divided into strong $(>75)(n=280)$ and weak $(<57)(n=433)$ SOC samples.

RESULTS: The strong SOC sample reported lower levels of anxiety $(p<0.001)$ and higher levels on functional health status $(p<0.001)$ at T1 and T2, compared to the weak SOC sample. Baseline anxiety predicted low functional health status in the weak SOC sample at T2. Anxiety had less negative consequences for function after rehabilitation in the strong SOC compared to the weak SOC sample.
\end{abstract}

CONCLUSIONS: The data suggest that appropriate interventions would strengthen the level of SOC in the most vulnerable, as well as resources that encourage SOC.

Keywords: Occupational disability, negative emotions, coping, multidisciplinary rehabilitation

\section{Introduction}

Chronic musculoskeletal pain is a prevalent problem with widespread negative economic, psychological, and social consequences for individuals as well as for labour and welfare organisations [1,2]. In the past

* Corresponding author: Monica Lillefjell, Department of Occupational Therapy, Faculty of Health Education and Social Work, University College of Sør-Trøndelag, Tungasletta 2, N-7046 Trondheim, Norway. Tel.: +47907765 70; E-mail: monica.lillefjell@ hist.no. decades, chronic musculoskeletal pain has become one of the leading causes of occupational disability in Norway, giving rise to concern [3].

Chronic widespread pain conditions (pain of $>$ 3 months duration) without any manifest organic disease, recently termed "medically unexplained symptoms" (MUS) [4], are viewed as a multidimensional phenomenon, characterized by considerable functional limitations and increasing health care consumption [2, 5-8]. A range of negative emotions, including anxiety, are frequently experienced by people coping with a va- 
Table 1

Baseline characteristics of study sample (\% or Mean/ SD)

\begin{tabular}{|c|c|c|}
\hline Characteristics & $\begin{array}{l}\text { Strong SOC sample } \\
n=280\end{array}$ & $\begin{array}{l}\text { Weak SOC sample } \\
n=433\end{array}$ \\
\hline Age (Mean/SD) & $50.5(8.9)$ & $43.9(9.5)^{* * *}$ \\
\hline Gender ( $\%$ females $)$ & 77.5 & $73.2^{*}$ \\
\hline Family situation (\% single, solitary) & 26.8 & $38.1^{* * *}$ \\
\hline \multicolumn{3}{|l|}{ Education level: $(\%)$} \\
\hline Primary school & 23.7 & 22.2 \\
\hline Technical/vocational school 1-2 year & 25.9 & 24.0 \\
\hline High school & 27.0 & $33.1^{* *}$ \\
\hline College/university $>4$ years & 21.2 & $15.2^{* *}$ \\
\hline College/university $<4$ years & 2.2 & 5.1 \\
\hline \multicolumn{3}{|l|}{ Present/last held job: $(\%)$} \\
\hline Unskilled & 21.1 & 21.3 \\
\hline Skilled worker/craftsman & 26.6 & $18.7^{* *}$ \\
\hline Driver, Manager owner, forest owner, Farmer & 8.0 & 4.8 \\
\hline Employee, official, officer, salaried employee & 19.6 & 18.1 \\
\hline Senior position/job/post (private or governmental) & 4.6 & 2.9 \\
\hline
\end{tabular}

riety of chronic pain conditions $[9,10]$. The relevance of sense of coherence, described as the dimensions of a person's response to a stressful situation [11], in the prediction of pain, has been shown in previous research [12,13]. Eriksson [14] suggests that a strong sense of coherence, improves the prospects that one will be able to cope with health problems. Efficacy in managing own health problems is also found to affect work adjustment in persons with chronic disease [15].

The theory of salutogenesis, introduced by the American-Israeli medical sociologist Aron Antonovsky, suggests that health should be seen as a continuum ranging from "total ill health" to "total health" $[11,16]$. Introducing the salutogenic concept of a "sense of coherence" (SOC) as the experience of one's life as comprehensible, manageable, and meaningful, he considered SOC to be closely linked to people's capacities for enjoying good health and coping with health problems [17-19]. Moreover, Antonovsky [16] considered factors protective of health to be qualitatively different from risk factors for health problems.

Strong SOC is regarded as a characteristic that helps people identify, use, and reuse available resources and therefore minimize feelings of tension and stress [20]. Focusing on the person's own experiences, Antonovsky stated [11] that persons with a strong sense of coherence will maintain positive health and well-being even in stressful situations. In contrast, a weak SOC is found to increase the likelihood of continued pain and functional problems [21].

The stability of one's sense of coherence has been widely discussed, and the assumption that SOC increases with age and changes as a result of major life events has been confirmed [22,23]. Stressful life events such as unemployment and financial difficulties have been shown to weaken SOC [23,24], whereas positive life events, such as recovery from serious illness or positive changes in one's working life, are associated with an increase in SOC [25].

Sense of coherence is found to be a significant moderator of the effects of stressful life events on health [26], and it is also suggested that the meaningfulness sub-scale, defined by Antonovsky [16], will significantly predict how people with chronic pain report their pain levels six months after a rehabilitation course [27]. Holmberg, Thelin and Stiernstrom [28] showed that SOC was strongly correlated to workrelated psychosocial factors and social support, independent of socio-demographic factors. In a systematic review of the literature, SOC was found to be strongly related to health, especially mental health [29]. High negative associations have been observed between the SOC scale and measures of anxiety, however previous research focusing on the relationship between the SOC scale and measures of negative emotions has yielded inconclusive results [30]. The existing literature suggests that more knowledge is required about the association between initial anxiety levels and physical functioning after rehabilitation in relation to individuals' own experiences of their health-resources, measured as sense of coherence at the beginning of the rehabilitation period.

The objective of the present study was to investigate the association between baseline anxiety and pain and functional health status after rehabilitation in employees who experience either a strong or weak sense of coherence and suffered from musculoskeletal pain during rehabilitation. 


\section{Methods}

\subsection{Design}

A prospective study of 1665 participants with chronic non-specific musculoskeletal pain conditions was conducted. All participants were volunteers and gave their informed consent. Confidentiality was emphasized. The study was approved by the Norwegian Social Science Data Service (NSD) and the Regional Committee for Medical and Health Research Ethics (REK) of Mid-Norway.

\subsection{Sample}

All participants, completing a multidisciplinary rehabilitation program at a private rehabilitation centre in Mid-Norway, were studied before and after the treatment period of 12 weeks. All participants $(N=1665)$, mean age $45.2 / \mathrm{SD}=9.1$, completed the treatment period, but results from two $(n=2)$ participants were rejected because of missing SOC questionnaires. Selfreported measures were used to collect demographic data and data on pain, functional health status, anxiety, and sense of coherence.

\subsection{Treatment program}

Based on a salutogenic approach [11,14,16,17], the multidisciplinary rehabilitation programme, developed for individuals on sick leave with complex musculoskeletal disease conditions, focuses strongly on strengthening existing resources; both internal and external, and allowing the participants to be aware of, identify, and benefit from these resources.

Participants were assigned to the rehabilitation programme by their GPs based on interviews, observations, and clinical tests. The participants attended the rehabilitation program approximately $6 \mathrm{~h}$ /day, $1-$ 4 days a week. All participants are matched with a personal supervisor, and individual counselling is offered during the rehabilitation period. Inclusion criteria for participation in the rehabilitation programme were that the participants received national insurance benefits in the form of a sickness benefit or rehabilitation benefit, and experienced mixed chronic pain problems. The rehabilitation program has a drop-out rate of approximately $6 \%$; the majority of the drop-outs are men (mean age 35 ).

\subsection{Measures and procedures}

SOC (13-item) [29], demographics, personal characteristics, pain intensity, pain experience (Visual Ana- logue Scale (VAS) [31], functional health status (COOP WONCA) [32], and anxiety (HADS) [33] were collected prior to treatment (T1) and after a 12-week rehabilitation period (T2).

The 13-item Norwegian version of the Sense of Coherence Scale (SOC), derived from a theoretical model designed to explain the maintenance or improvement of one's position on a health-ease/disease continuum, was utilized to measure the three main elements of comprehensibility, manageability, and meaningfulness. The SOC is a 7-point numeric scale, with higher scores indicating stronger sense of coherence. The validity and reliability of the scale have been tested in studies in more than 16 countries. Some of the studies reported test-retest correlations, which were stable at 0.54 over two years $[29,30,34]$. Examples of items are: "Do you have the feeling that you don't really care about what goes on around you?", "Has it happened that people whom you counted on disappointed you?", and "Doing the things you do every day is: A source of deep pleasure and satisfaction/A deep source of pain and boredom." Examples of the start and end points of the scale range from "Very seldom or never to Very often", and from "Never happened to Always happened". The internal consistency in the present study showed Cronbach's coefficient alpha of 0.82 for the total SOC.

The Visual Analogue Scale (VAS) [31,35] was employed to assess variables about pain (degree of pain and how troublesome you experience the pain to be). The use of the VAS in a pain-suffering population is well established, and has shown good reliability and validity, with significant correlation ranging from $r=$ $0.75-r=0.83[35,36]$. The scale is a line of $10 \mathrm{~cm}$ on which marks are scored in millimetres, representing the continuum of the symptom to be rated. Items included:

- Pain - On a scale from 0-100 where 0 is "no pain" and 100 is "unbearable pain," how much pain do you have at present?

- Pain experience - On a scale from 0-100 where 0 is "not troublesome at all" and 100 is "extremely troublesome," how troublesome do you experience the pain to be in everyday life?

Functional health status was measured using the Norwegian version of the COOP/WONCA Charts [31, 32]. The COOP/WONCA charts measure six core aspects of functional status; physical fitness, feelings, daily activities, social activities, changes in health and overall health. The instrument consists of six charts, referring to the above-mentioned aspects of functioning. Each chart consists of a simple title, a question re- 
ferring to the status of the patient and an ordinal fivepoint response scale illustrated with a simple drawing. Each item is rated on a five-point ordinal scale ranging from 1 ("no limitation at all") to 5 ("severely limited"). For "change in health," a score of 1 means "much better" and a score of 5 "much worse." The COOP/WONCA charts reflect the patient's assessment of his/her functional capacity at the given time. The test-retest reliability of the original Dartmouth version and the Norwegian version is satisfactory $(r=0.74$ 0.86 ) [31] and the subscales, such as function and feelings, have been reported to correlate well with other measures of physical and emotional functioning [35].

Anxiety was assessed using the Hospital Anxiety and Depression Scale (HADS) [31,37,38]. It is a brief assessment of anxiety and depression, consisting of 14 items divided into two sub-scales for anxiety (7 items) and depression (7 items), in which the patient rates each item on a four-point scale. Individual items are scored from 0-3 to 3-0, depending on the direction of the wording of the items. The scores of the items represent the degree of distress: $0=$ none, $1=$ a little, $2=$ a lot, 3 = unbearable. All items are summed. Higher scores indicate the presence of problems. Based on data from a large population, Mykletun et al. [33] suggest that the basic psychometric properties of the HAD scale as a self-rating instrument should be considered quite good in terms of factor structure, intercorrelation, homogeneity, and internal consistency. The internal consistency in the present study showed Cronbach's coefficient alpha of 0.82 for the entire HADS, as well as for the anxiety subscale.

\subsection{Statistical analysis}

Statistical analysis was carried out via SPSS/ PC+ version 17.0. Descriptive statistics of frequencies, percentages, mean values, and standard deviation were calculated for continuous and categorical variables in present sample. Internal consistency, Cronbach's coefficient alpha, was calculated for the HADS and the SOC scale. Based on validated cut-off values, the employees were divided into strong $(>75)(n=280)$ and weak $(<57)(n=433)$ SOC samples [14,29]. Those with SOC scores from 57-75 were excluded from further analyses.

$T$-tests were performed on the pain, anxiety, SOC, and functional health status variables to compare the means at the first and second measurement in the strong and weak SOC samples. Bivariate associations between the baseline variables in this study were per- formed using Persons $(r)$ correlation coefficient. Linear multiple regression analyses were used to evaluate the association between baseline anxiety and pain and functional health status (physical fitness, feelings, daily activities, social activities, changes in health, and overall health) measured at the end of the rehabilitation period in the strong and weak sense of coherence samples. Final analyses were controlled for gender and age. The predictor variables (gender and age, pain intensity and experience, and anxiety) were included simultaneously into the equation. Analyses were carried out separately to define outcome variables for functional health status (physical fitness, feelings, daily activities, social activities, change in health, and overall health). P-values $\leqslant 0.05$ were considered statistically significant.

\section{Results}

\subsection{Characteristics}

The baseline characteristics of the study participants are shown in Table 1 . The mean age is significantly lower in the weak SOC sample compared to the strong SOC sample. Moreover, the portion of people living alone is higher in the weak SOC sample compared to the strong SOC sample. Additionally, the weak SOC sample reported lower education levels than the strong SOC sample (Table 1).

\subsection{Differences between the weak SOC and strong SOC samples}

Differences between the weak SOC $(<57)(n=$ $433)$ and the strong SOC $(>75)(n=280)$ samples in terms of pain, anxiety, and functional health status reported at baseline (T1) and at the end of the rehabilitation period (T2) are shown in Table 2. The strong SOC sample reported higher levels of pain intensity and experienced the pain as more troublesome at baseline compared to the weak SOC sample. However, the mean scores of pain intensity and how troublesome the pain is experienced to be decrease more sharply between $\mathrm{T} 1$ and $\mathrm{T} 2$ in the strong SOC sample than do those in the weak SOC sample.

The weak SOC sample reported significantly $(p<$ 0.001 ) higher levels of anxiety at both T1 and T2 than did the strong SOC sample. While both SOC samples' mean anxiety scores decreased from $\mathrm{T} 1$ to $\mathrm{T} 2$, those with weak SOC still reported anxiety levels above 
Table 2

Differences between the weak SOC $(<57)(n=433)$ and the strong SOC $(>75)(n=280)$ samples on pain, anxiety and functional health status (physical fitness, feelings, daily activities, social activities, change in health and overall health) at baseline (T1) and at the end of the rehabilitation (T2) (Mean/SD, t-value, p-value) (t-test)

\begin{tabular}{|c|c|c|c|c|}
\hline & Weak SOC $n=433$ & Strong SOC $n=280$ & $\mathrm{~T}$ & $\mathrm{P}$ \\
\hline Pain intensity (T1) & $66.3 / 24.3$ & $71.0 / 23.0$ & 3.40 & 0.001 \\
\hline Pain intensity(T2) & $63.5 / 23.6$ & $64.2 / 27.7$ & 0.40 & 0.689 \\
\hline Pain experience (T1) & $60.8 / 27.9$ & $66.2 / 26.9$ & 3.36 & 0.001 \\
\hline Pain experience (T2) & $54.3 / 26.2$ & $56.7 / 28.6$ & 1.29 & 0.197 \\
\hline Anxiety (T1) & $12.3 / 4.2$ & $4.0 / 2.9$ & -46.6 & 0.000 \\
\hline Anxiety (T2) & $9.1 / 5.2$ & $3.1 / 2.8$ & -34.9 & 0.000 \\
\hline Physical fitness (T1) & $2.63 / .99$ & $2.64 / 1.0$ & 0.21 & 0.827 \\
\hline Physical fitness (T2) & $2.55 / .99$ & $2.62 / 1.0$ & 0.98 & 0.323 \\
\hline Feelings(T1) & $3.95 / 0.86$ & $1.95 / 0.92$ & -35.90 & 0.000 \\
\hline Feelings(T2) & $3.31 / .1 .0$ & $1.80 / .93$ & -24.39 & 0.000 \\
\hline Daily activities (T1) & $3.33 / 0.80$ & $2.83 / 1.0$ & -7.80 & 0.000 \\
\hline Daily activities (T2) & $2.82 / .99$ & $2.59 / 1.0$ & -3.30 & 0.001 \\
\hline Social activities (T1) & $3.22 / 1.0$ & $1.96 / 1.1$ & -19.00 & 0.000 \\
\hline Social activities (T2) & $2.59 / 1.1$ & $1.76 / 95$ & -13.25 & 0.000 \\
\hline Changes in health (T1) & $1.95 / 0.87$ & $1.24 / 0.51$ & -23.25 & 0.000 \\
\hline Changes in health (T2) & $1.76 / 0.82$ & $1.23 / 0.50$ & -16.05 & 0.000 \\
\hline Overall health (T1) & $3.60 / 0.67$ & $2.85 / 0.83$ & -15.18 & 0.000 \\
\hline Overall health (T2) & $3.06 / 0.82$ & $2.60 / 0.84$ & -8.19 & 0.000 \\
\hline
\end{tabular}

the validated cutoff value $(\geqslant 8)$ at $\mathrm{T} 2$. Additionally, the strong SOC sample was characterized by significantly greater functional health status on all six core aspects of functioning measured by the COOP/WONCA (physical fitness, feelings, daily activities, social activities, changes in health and overall health) at baseline as well as at the end of the rehabilitation period.

\subsection{Correlations between age, gender, pain, anxiety, and functional health status in the strong and weak SOC samples}

The results of the correlation analysis of the variables included in the study are presented in Table 3. High levels of anxiety were associated with limitations in functioning on all core aspects of functional health status except physical functioning in the weak SOC sample.

Anxiety correlated negatively with pain intensity in the strong SOC sample. Moreover, in the strong SOC sample anxiety was associated with limitation in functioning on the following core aspects: emotional functioning (feelings), social activities, change in health, and overall health in.

\subsection{Regression analysis for variables predicting functional health status}

Table 4 presents the results of multiple linear regression analysis investigating the association between baseline age, gender, pain, and anxiety and the post treatment outcome of functional health status (physical fitness, feelings, daily activities, social activities, and overall health) in the weak SOC sample. For the purpose of present analysis, there exists no definitive theoretical justification for ordering the entry of variables, so we opted for the simultaneous entry of all variables.

\subsubsection{Physical fitness}

All together the variables entered into the equation explained $8 \%$ of the variance in physical fitness, but only age and pain experience contributed significant part correlations. Pain $(p<0.01)$ and age $(p<0.05)$ together explained $5 \%$ of the variable variance in the weak SOC sample.

\subsubsection{Feelings}

All of the variables in the regression equation contributed to explain $5 \%$ of the variance in emotional feelings, with anxiety $(p<0.001)$ contributing the only significant individual part.

\subsubsection{Daily activities}

All together the variables entered into the equation explained $11 \%$ of the variance in daily activities. Only pain experience $(p<0.001)$ and anxiety $(p<0.001)$ contributed significant part correlations.

\subsubsection{Social activities}

High levels of anxiety $(p<0.001)$ were found to be significantly associated with limitations in function in social activities. All together the variables entered into the equation explained $9 \%$ of the variance. 
Table 3

Correlations between age, gender, pain intensity, pain experience, anxiety and functional health status (physical fitness, feelings, daily activities, social activities, change in health and overall health) in the strong and weak sense of coherence (SOC) samples

\begin{tabular}{|c|c|c|c|c|c|c|c|c|c|c|c|}
\hline & $\mathrm{a}$ & $\mathrm{b}$ & $\mathrm{c}$ & $\mathrm{d}$ & $\mathrm{e}$ & f & g & $\mathrm{h}$ & $\mathrm{i}$ & $\mathrm{j}$ & $\mathrm{K}$ \\
\hline $\operatorname{Age}^{a}$ & - & -0.01 & $-0.18^{* *}$ & -0.11 & 0.07 & 0.09 & 0.03 & 0.07 & -0.01 & 0.02 & 0.05 \\
\hline Gender $^{\mathrm{b}}$ & $0.10^{*}$ & - & 0.03 & -0.01 & -0.08 & 0.03 & -0.10 & 0.05 & $-0.16^{*}$ & 0.06 & -0.00 \\
\hline Pain intensity ${ }^{\mathrm{c}}$ & -0.01 & -0.01 & - & $0.74^{* *}$ & $-0.15^{*}$ & $0.13^{*}$ & $-0.16^{* *}$ & $0.31^{* *}$ & 0.07 & -0.10 & $0.14^{*}$ \\
\hline Pain experience ${ }^{\mathrm{d}}$ & -0.08 & 0.04 & $0.76^{* *}$ & - & -0.05 & $0.15^{*}$ & -0.09 & $0.39^{* *}$ & $0.14^{*}$ & -0.07 & $0.22^{* *}$ \\
\hline Anxiety $^{\mathrm{e}}$ & -0.01 & 0.06 & -0.06 & -0.03 & - & -0.05 & $0.53^{* *}$ & 0.08 & $0.22^{* *}$ & $0.31^{* *}$ & $0.22^{* *}$ \\
\hline Physical fitness ${ }^{\mathrm{f}}$ & $0.14^{* *}$ & -0.06 & $0.14^{* *}$ & $0.18^{* *}$ & -0.01 & - & 0.02 & $0.29^{* *}$ & $0.15^{* *}$ & -0.08 & $0.23^{* *}$ \\
\hline Feelings $\mathrm{g}$ & -0.05 & 0.00 & -0.08 & $-0.13^{* *}$ & $0.49^{* *}$ & -0.01 & - & 0.05 & $0.21^{* *}$ & $0.23^{* *}$ & $0.28^{* *}$ \\
\hline Daily activities ${ }^{h}$ & 0.02 & 0.08 & $0.12^{* *}$ & 0.19 & $0.18^{* *}$ & $0.17^{* *}$ & $0.25^{* *}$ & - & $0.47^{* *}$ & -0.02 & $0.48^{* *}$ \\
\hline Social activities ${ }^{\mathrm{i}}$ & 0.00 & 0.02 & -0.02 & -0.06 & $0.37^{* *}$ & 0.06 & $0.39^{* *}$ & $0.38^{* *}$ & - & -0.00 & $0.38^{* *}$ \\
\hline Change in health ${ }^{\mathrm{j}}$ & 0.09 & $0.17^{* *}$ & -0.04 & -0.03 & $0.39^{* *}$ & -0.00 & $0.28^{* *}$ & $0.23^{* *}$ & $0.30^{* *}$ & - & 0.09 \\
\hline Overall health ${ }^{\mathrm{k}}$ & -0.06 & $0.09^{*}$ & 0.08 & 0.08 & $25^{* *}$ & 0.09 & $0.35^{* *}$ & $0.35^{* *}$ & $0.32 * *$ & $0.22 * *$ & - \\
\hline Cronbach's $\alpha$ & - & - & - & - & 0.82 & - & - & - & - & - & - \\
\hline
\end{tabular}

Note. ${ }^{*} p \leqslant 0.05{ }^{* *} p \leqslant 0.01$. Correlations for the weak SOC sample are below the diagonal and correlations for the strong SOC sample are above the diagonal.

\subsubsection{Change in health}

The variables entered into the equation together explained $18 \%$ of the variance in one's experience of positive change in health during the last 2 weeks. Only gender (female) $(p<0.001)$, and low levels of anxiety $(p<0.001)$, contributed significant part correlations.

\subsubsection{Overall health}

Limitation in overall health at the end of the rehabilitation program in the weak SOC sample was associated with baseline pain experience $(p<0.01)$ and high levels of anxiety $(p<0.001)$. All together the variables entered into the equation explained $7 \%$ of the variance.

Table 5 presents the results of the multiple linear regression analysis investigating the association between baseline age, gender, pain, and anxiety and the posttreatment outcome of functional health status (physical fitness, feelings, daily activities, social activities, and overall health) in the strong SOC sample. As described above we opted for the simultaneous entry of all variables.

\subsubsection{Physical fitness}

Results of the regression analysis showed that the variables entered explained $11 \%$ of the variance in physical fitness. Only age $(p<0.001)$ contributed significant part correlation.

\subsubsection{Feelings}

The variables entered into the equation together explained $14 \%$ of the variance in emotional functioning (feelings). Gender (female) $(p<0.02)$ and high levels of anxiety $(p<0.001)$ contributed significant part correlations.

\subsubsection{Daily activities}

All together the variables entered explained $24 \%$ of the variance in daily activities. High age $(p<0.05)$, as well as high levels of pain intensity $(p<0.01)$ and pain experience $(p<0.05)$ contributed significant part correlations. Anxiety was not significantly associated with function in daily activities in the strong SOC sample.

\subsubsection{Social activities}

The variables entered in the regression equation contributed to explain $9 \%$ of the variance in social activities in the strong SOC sample. Only high levels of anxiety $(p<0.001)$ contributed significant part correlation.

\subsubsection{Change in health}

The variables entered in the equation explained $5 \%$ of the variance in change in health, and gender (female) $(p<0.001)$ represented the only significant part correlation.

\subsubsection{Overall health}

In total the variables entered in the equation model explained $15 \%$ of the variance in overall health. Whereas high age $(p<0.01)$ and baseline pain intensity $(p<0.05)$ contributed with significant part correlations. Anxiety was not a significant associated with limitation in functioning in the strong SOC sample.

\section{Discussion}

This study of a Norwegian sample suggests that the association between anxiety and pain level at baseline 
M. Lillefjell et al. / The impact of a sense of coherence in employees with chronic pain

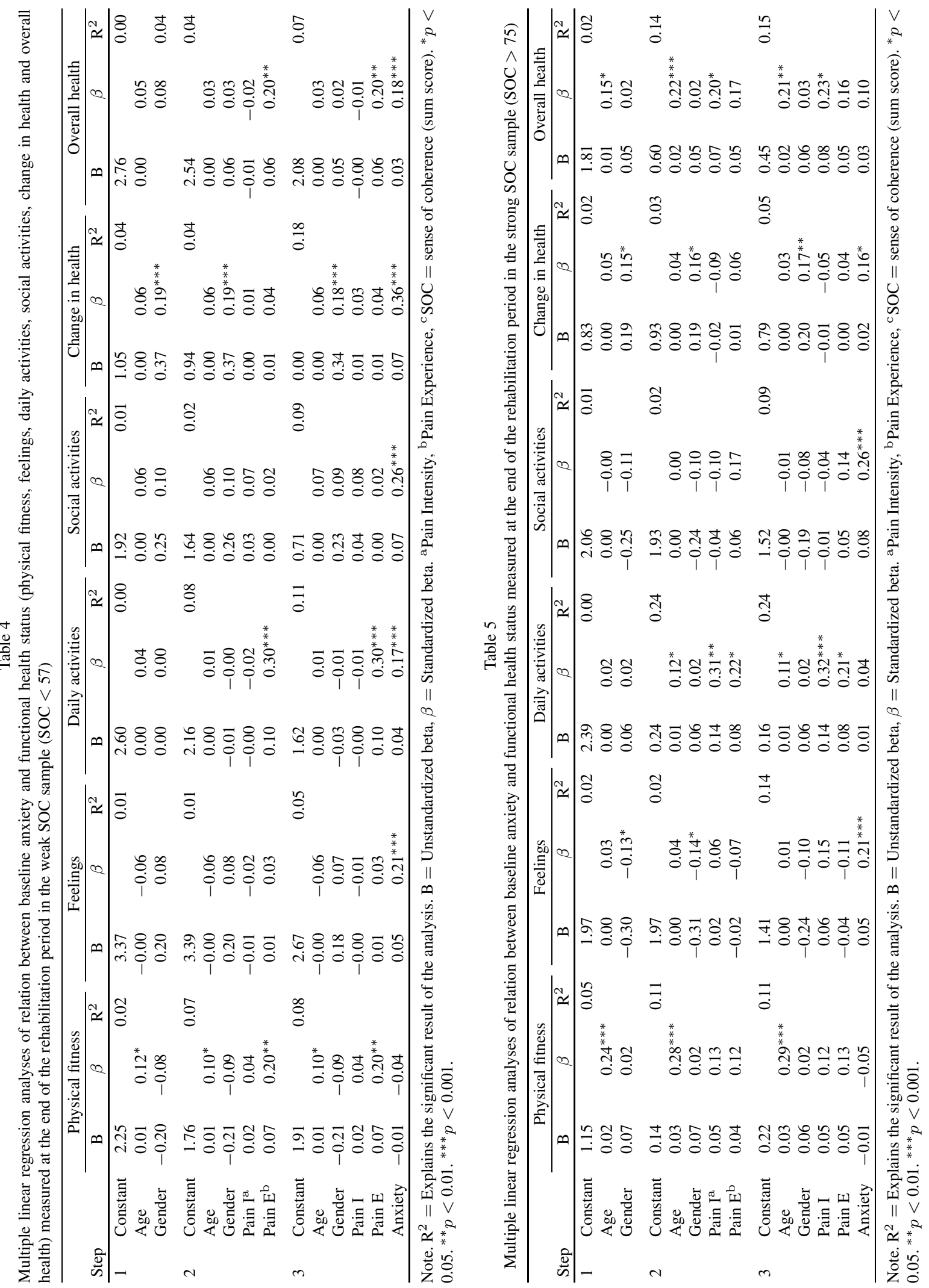


and functional health after rehabilitation is significantly stronger among persons with non-specific musculoskeletal pain and a weak sense of coherence at baseline than among those with a baseline strong sense of coherence.

Previous studies have indicated an association between levels of SOC and anxiety in predicting functional health status [7,8,20,21]. A strong SOC has been identified as helpful in allowing people to identify, use, and reuse available resources and consequently minimize feelings of tension and stress [20]. In this study, compared to the weak SOC sample, the strong SOC sample reported significantly higher levels of pain intensity and experienced the pain as more troublesome at baseline as well as at the end of the rehabilitation period. However, in support of the salutogenic concept, which proposes that SOC is closely linked with an individual's resources and capacity to create health and cope with health problems [Antonovsky 17, 19, 20], the strong SOC sample, despite significant pain, reported higher function on all six core aspects of functional health at the end of the rehabilitation program. Additionally, the change in mean scores in pain intensity and how troublesome the pain is experienced to be from baseline to the end of the treatment period were larger in the strong SOC sample.

People coping with a variety of chronic pain conditions routinely experience emotions such as anxiety $[9,10]$. Our high SOC sample reported significantly higher levels of pain then the weak SOC sample. However, the strong SOC sample reported almost no anxiety whatsoever. In support of previous studies [14,16], this result might indicate that a strong sense of coherence improves one's ability to deal with health problems. One's level of SOC and level of anxiety can be a product of the time it takes to seek care and start the recovery process. Given that those with a strong SOC also have higher levels of education than those with weak SOC, it is conceivable that this affects levels of SOC and anxiety. Those with higher levels of education are used to managing systems and finding and utilizing available information, and are thus able to enter the rehabilitation process far more quickly than those with less education.

Since the weak SOC sample showed less improvement in function from baseline to the end of the treatment period than the strong SOC sample, it is reasonable to assume that a weak SOC and high levels of anxiety increase the likelihood of sustained pain and functional problems. This is in line with previous findings suggesting that a weak SOC increases the odds of sus- tained pain and functional problems [21]. The results therefore present implications for the design of rehabilitation programs, and encourage further clarification of the role of sense of coherence and the use of sense of coherence in the rehabilitation context to minimize feelings of tension and to improve function in daily life [20].

Due to the limitations of our study design, we must also be open to the possibility that measures to reduce anxiety could also lead to increased SOC. As the rehabilitation period was limited to 12 weeks, it is likely that minimal changes in SOC occurred during the rehabilitation period. Reduction of anxiety in individuals with weak SOC at the start of any recovery would, however, be an important step towards the improvement of several other functional areas.

Additionally, the results revealed the significance of anxiety in predicting functional health status in both the strong and weak SOC samples. Although anxiety was the most significant predictor of function in both samples, the results indicate that it is important to increase levels of SOC, as well as resources that encourage SOC, in those who are most vulnerable [20]. Since anxiety had a less negative effect on the strong SOC sample, it would likely be useful if rehabilitation counsellors adopted a systematic salutogenic orientation to focus on the resources that are available. Strengthening cognitive as well as emotional resources, creating new ones, and making them known to the individuals so that they may be aware of, identify, and benefit from them will, however, require these professionals to possess adequate knowledge and employ adequate methods of intervention $[11,14]$.

\subsection{Limitations}

The present study suffers from a few limitations. In this follow-up study, the employee who had mixed pain diagnoses did not receive identical standardized interventions, as would be expected in randomized controlled trials [39]. However, the aims of this study were to examine the characteristics of individuals in a real clinical setting and determine how these characteristics might affect function in daily life. The repeated measures performed in the present study mean that the observations are dependent, and this might cause incidental effects like order effects (practice), carry-over effects, and context effects [39]. Measurement itself may influence the outcome by inducing efforts to address a particular risk factor.

Another limitation of these results is the possibility of bias that accompanies self-reporting. Psychoso- 
cial factors like emotional distress, secondary loss, somatisation and symptom magnification, compliance, and resistance issues might have influenced the selfassessments [9]. Additionally, differences in age, education and family situation, between the high and low SOC groups, might have influenced the results since these background factors may be related to levels of SOC.

Despite these shortcomings, the present study does raise some interesting perspectives on SOC, anxiety, and function in daily life that should be taken into account in rehabilitation settings.

\subsection{Conclusion}

In conclusion, the data suggest that it would be beneficial to reduce anxiety in those with weak SOC and a high degree of anxiety. Additionally, more research is necessary: both longitudinal investigations of factors that appear to strengthen SOC in rehabilitation programs for employees with chronic musculoskeletal pain, as well as research that seeks to examine the extent to which an improvement in function can be understood as a result of enhanced SOC or absence/reduction of anxiety, or both.

\section{Acknowledgement}

The authors would like to thank the participants and Friskgården for participation in this study.

\section{References}

[1] Hagen K, Zwart JA, Svebak S, Bovim G, Stovner LJ. Low socioeconomic status is associated with chronic musculoskeletal complaints among 46,901 adults in Norway. Scand J Public Health 2005; 33: 268-275.

[2] Breivik H, Collett B, Ventafridda V, Cohen R, Gallacher D. Survey of chronic pain in Europe: prevalence, impact on daily life, and treatment. Eur J Pain. 2006; 10(4): 287-333.

[3] Statistics Norway. www.ssb.no. 21.12.12.

[4] Tschudi-Madsen H, Kjeldsberg M, Natvig B, Ihlebaek C, Dalen I, Kamaleri Y,Bruusgaard D. A Strong Association Between Non-musculoskeletal Symptoms and Musculoskeletal Pain Symptoms. Results from a Population Study. BMC Musculoskelet Disord 2011; 12(285).

[5] Trunchon M. Determinants of chronic disability related to low back pain: Towards a integrative biopsychosocial model. Disabil Rehabil 2001; 23: 758-767.

[6] McCracken LM, Turk DC. Behavioural and cognitivebehavioural treatment for chronic pain: outcome, predictors of outcome, and treatment. Spine 2002; 22: 2564-73.
[7] Lillefjell M, Krokstad S, Espnes GA. Prediction of function in daily life following multidisciplinary rehabilitation for individuals with chronic musculoskeletal pain; A prospective study.BMC Musculoskeletal Disorders 2007; 8: 65.

[8] Lillefjell M. Function and Work Ability Following Multidisciplinary Rehabilitation for Individuals with Chronic Musculoskeletal Pain. Doctoral theses, NTNU, 2008: 184.

[9] Gatchel R, Peng Y, Peters M, Fuchs P, Turk D. The biopsychosocial approach to chronic pain: Scientific advances and future directions. Psychol Bull 2007; 133(4): 581-624.

[10] Thompson M, McCracken LM. Acceptance and related processes in adjustment to chronic pain. Curr Pain Headache Rep 2011; 15: 144-151.

[11] Antonovsky A. Health, Stress and Coping. New Perspectives on Mental and Physical Well-being. San Francisco: JosseyBass publishers, 1979.

[12] Schult ML, Söderback I, Jacobs K. (2000). The sense-ofcoherence and the capability of performing daily occupations in persons with chronic pain. Work 2000; 15: 189-201.

[13] Lillefjell M, Jakobsen K. Sense of Coherence as a Predictor of Work Reentry Following Multidisciplinary Rehabilitation for Individuals With Chronic Musculoskeletal Pain. J Occup Health Psychol 2007; 12 (3): 222-231.

[14] Eriksson M. Unravveling the mystery of salutogenesis. The ecidenc base of the salutogenic research as measured by Antonovsky's Sense of Cohernece Scale. Research Report. Turku. Åbo Akademis Tryckeri, 2007.

[15] Siu AMH, Hung A, Lam AYL, Cheng A. Work limitations, workplace concerns, and job satisfaction of persons with chronic disease. Work 2013; 45: 107-115.

[16] Antonovsky A. Unravelling the mystery of health - How people manage stress and stay well. San Francisco: Jossey-Bass publishers, 1987.

[17] Antonovsky A. The structure and properties of the sense of coherence scale. Soc Sci Med 1993; 36: 725-733.

[18] Volanen SM, Lahelma E, Silventoinen K, Suominen S. Factors contributing to sense of coherence among men and women. Eur J Publ Health 2004;14: 322-330.

[19] Eriksson M, Lindström B, Lilja JA. Sense of coherence and health. Salutogenesis in a societal context: Aland, a special case? J Epidemiol Community Health 2007; 61(8): 684-8.

[20] Volanen SM, Suominen S, Lahelma E, Koskenvuo K, Koskenvuo M, Silventoinen K. Sense of coherence and intentions to retire early among Finnish women and men BMC Public Health 2010;10: 22, p. 12.

[21] Suominen S, Gould R, Ahvenainen J, Vahtera J, Uutela A, Koskenvuo M. Sense of coherence and disability pension. A nationwide, register based prospective population study of 2196 adult Finns. J Epidemiol Community Health 2005; 59: 455-459.

[22] Nilsson B, Holmgren L, Stegmayr B, Westman G. Sense of coherence - stability over time and relation to health, disease, and psychosocial changes in a general populati on: alongitudinal study. Scand J Public Health 2003; 31: 297-304.

[23] Feldt E, Leskinen E, Kinnunen U. Structural invariance and stability of sense of coherence: A longitudinal analysis of two groups with different employment experiences. Work Stress 2005; 19, 68-83.

[24] Paul KI, Moser K. 'Incongruence as an explanation for the negative mental health effects of unemployment: Metaanalytic evidence'.J Occup Organ Psychol 2006; 79: 595-621

[25] Kivimäki M, Feldt T, Vahtera J, Nurmi JE. Sense of coherence and health: Evidence from two cross-lagged longitudinal samples. Social Sci Med 2000; 50: 583-597. 
[26] Richardson CG, Ratner P. Sense of coherence as a moderator of the effects of stressful life events on health. J Epidemiol Community Health 2005; 59: 979-984.

[27] Callahan L, Pincus T. The Sense of Coherence Scale in patients with rheumatoid arthritis. Arthritis Care Research 1995; 8: 28-35.

[28] Holmberg S, Thelin A, Stiernstrom EL Relationship of sense of coherence to other psychosocial indices. Eur J Psychol Assess 2004; 20: 227-236 7.

[29] Eriksson M, Lindström B. Validity of Antonovsky's sense of coherence scale: A systematic review. J Epidemiol Community Health, 2005; 59: 460-466.

[30] Konttinen H, Haukkala A, Uutela A. Comparing sense of coherence, depressive symptoms and anxiety, and their relationships with health in a population-based study Soc Sci Med 2008; 2401-2412.

[31] Bowling A. Measuring Health. A review of quality of life measurement scales. Second edition, Buckingham and Philadelphia: Open University Press, 1997.

[32] Bentsen BG, Natvig B,Winnem M. Assesment of own functional capacity. COOP-WONCA Charts in clinical work and research. Tidsskrift Norske Lægeforening 1997; 117: 545549
[33] Mykletun A, Stordal E, Dahl AA. Hospital anxiety and depression (HAD) scale: factor structure, its analyses and internal consistency in a large population. British Journal of Psychiatry $2001 ; 179$ : 540-544.

[34] Smith PM, Breslin FC, Beaton DE. Questioning the stability of sense of coherence. The impact of socio-economic status and working condition in the Canadian population. Social Psychiatry Psychiatric Epidemiology, 2003; 38: 475-484.

[35] McDowell I, Newell C. Measuring Health - A Guide to Rating Scales and Questionaires. New York: Oxford University Press, 1997.

[36] Bergh I. Assessing pain in elderly. Rating scales, and their relation to verbal expression of pain and pain relief. Unpublished Licentiate thesis. Sweden: Gøteborg University, 2001.

[37] Zigmond AS, Snaith RP. The hospital anxiety and depression scale. Acta Psychiatrica Scandinavica 1983; 67: 361-370.

[38] Snaith RP. The Hospital Anxiety and Depression Scale. Health Qual Life Outcomes 2003; 1: 29.

[39] Keppel G, Wickens TD. Design and Analysis: A Researcher's Handbook. Prentice Hall, 2004. 